\title{
CORRECTION
}

\section{Correction to: Food insecurity as a determinant of international migration: evidence from Sub-Saharan Africa}

\author{
Ahmad Sadiddin ${ }^{1} \cdot$ Andrea Cattaneo $^{1} \cdot$ Marinella Cirillo $^{1} \cdot$ Meghan Miller $^{1}$ \\ Published online: 4 March 2022 \\ (c) International Society for Plant Pathology and Springer Nature B.V. 2022
}

Correction to: Food Security (2019) 11: 515-530

https://doi.org/10.1007/s12571-019-00927-w

There is a typo in Table 1 where all values in parentheses, which report the standard error must be positive. The correct Table 1 is now given below.

Table 1 Average marginal effects and conditional average marginal effects from three binary-choice econometric equations

\begin{tabular}{|c|c|c|c|}
\hline Variables & Migration desire & Migration planning & Migration decision \\
\hline \multirow[t]{2}{*}{ Moderate Food insecurity } & $0.039 * * * *$ & -0.013 & $-0.069 * *$ \\
\hline & $(0.050)$ & $(0.090)$ & $(0.200)$ \\
\hline \multirow[t]{2}{*}{ Severe Food insecurity } & $0.052 * * * *$ & 0.017 & $-0.096^{* * *}$ \\
\hline & $(0.050)$ & $(0.090)$ & $(0.20)$ \\
\hline \multirow[t]{2}{*}{ Rural area } & $-0.050 * * * *$ & $-0.028 * *$ & -0.020 \\
\hline & $(0.050)$ & $(0.070)$ & $(0.100)$ \\
\hline \multirow[t]{2}{*}{ Age } & $-0.004 * * *$ & $0.007 * * * *$ & $0.003 * *$ \\
\hline & $(0.009)$ & $(0.010)$ & $(0.007)$ \\
\hline \multirow[t]{2}{*}{ Age squared } & -0.000 & $-0.000 * * * *$ & 0.000 \\
\hline & $(0.000)$ & $(0.000)$ & $(0.000)$ \\
\hline \multirow[t]{2}{*}{ Gender male } & $0.050 * * * *$ & -0.010 & 0.017 \\
\hline & $(0.040)$ & $(0.060)$ & $(0.100)$ \\
\hline \multirow[t]{2}{*}{ Log household income } & $0.020^{* * *}$ & -0.002 & 0.002 \\
\hline & $(0.040)$ & $(0.080)$ & $(0.200)$ \\
\hline \multirow[t]{2}{*}{ Married or domestic partner } & $-0.049 * * * *$ & -0.016 & 0.010 \\
\hline & $(0.040)$ & $(0.080)$ & $(0.200)$ \\
\hline \multirow[t]{2}{*}{ Employed part time } & $0.020 * *$ & 0.020 & 0.020 \\
\hline & $(0.050)$ & $(0.080)$ & $(0.200)$ \\
\hline \multirow[t]{2}{*}{ Employed full time } & $0.059^{* * * *}$ & $0.038 * * *$ & 0.008 \\
\hline & $(0.050)$ & $(0.080)$ & $(0.200)$ \\
\hline \multirow[t]{2}{*}{ Number of children } & -0.002 & 0.020 & 0.050 \\
\hline & $(0.010)$ & $(0.090)$ & $(0.200)$ \\
\hline
\end{tabular}

The original article can be found online at https://doi.org/10.1007/ s12571-019-00927-w.

Ahmad Sadiddin

ahmad.sadiddin@fao.org

1 Food and Agriculture Organization of the United Nations, Rome, Italy 
Table 1 (continued)

\begin{tabular}{|c|c|c|c|}
\hline Variables & Migration desire & Migration planning & Migration decision \\
\hline \multirow[t]{2}{*}{ Number of adults } & 0.003 & 0.002 & -0.007 \\
\hline & $(0.050)$ & $(0.050)$ & $(0.100)$ \\
\hline \multirow[t]{2}{*}{ Household size } & $0.005^{* * * *}$ & 0.002 & 0.001 \\
\hline & $(0.007)$ & $(0.010)$ & $(0.030)$ \\
\hline \multirow[t]{2}{*}{ Secondary education } & $0.059 * * * *$ & $0.021 *$ & 0.019 \\
\hline & $(0.040)$ & $(0.080)$ & $(0.200)$ \\
\hline \multirow[t]{2}{*}{ Post-secondary education } & $0.079 * * * *$ & $0.056^{* *} *$ & 0.080 \\
\hline & $(0.100)$ & $(0.140)$ & $(0.300)$ \\
\hline \multirow[t]{2}{*}{ Importance of religion } & -0.008 & -0.013 & -0.075 \\
\hline & $(0.070)$ & $(0.130)$ & $(0.200)$ \\
\hline \multirow[t]{2}{*}{ Support from abroad } & $0.102 * * * *$ & $0.120 * * * *$ & $0.190 * * * *$ \\
\hline & $(0.040)$ & $(0.080)$ & $(0.200)$ \\
\hline \multirow[t]{2}{*}{ Internet access } & $0.027^{* *} *$ & -0.003 & -0.026 \\
\hline & $(0.072)$ & $(0.100)$ & $(0.200)$ \\
\hline \multirow[t]{2}{*}{ Satisfaction with education system } & $-0.021 * * *$ & $-0.018^{*}$ & -0.012 \\
\hline & $(0.003)$ & $(0.070)$ & $(0.100)$ \\
\hline \multirow[t]{2}{*}{ Satisfaction with access to water } & -0.010 & 0.001 & 0.007 \\
\hline & $(0.040)$ & $(0.070)$ & $(0.100)$ \\
\hline \multirow[t]{2}{*}{ Overall satisfaction with city or area of residence } & $-0.095^{* * * *}$ & -0.017 & 0.009 \\
\hline & $(0.040)$ & $(0.070)$ & $(0.100)$ \\
\hline \multirow[t]{2}{*}{ Satisfaction with healthcare } & -0.004 & 0.002 & 0.028 \\
\hline & $(0.040)$ & $(0.080)$ & $(0.100)$ \\
\hline \multirow[t]{2}{*}{ Overall Satisfaction with standards of living } & $-0.020 * * *$ & 0.013 & $0.055^{* *}$ \\
\hline & $(0.040)$ & $(0.080)$ & $(0.100)$ \\
\hline Pseudo-R-squared (Conditional) & 0.270 & 0.140 & 0.220 \\
\hline Number of observations & 29,492 & 7,732 & 1,525 \\
\hline
\end{tabular}

Coefficients are average marginal effects for migration desire, and average conditional marginal effects for migration planning and preparations (decisions). Standard errors are shown in parentheses. Each equation includes country fixed effects

* Significant at the 10 percent level; ** Significant at the 5 percent level; *** Significant at the 1 percent level; **** Significant at the 0.1 percent level 